\title{
A tunable narrowband entangled photon pair source for resonant single-photon single-atom interaction
}

\author{
Albrecht Haase, Nicolas Piro, Jürgen Eschner*, and Morgan W. Mitchell \\ ICFO - Institut de Ciencies Fotoniques, \\ Mediterranean Technology Park, 08860 Castelldefels (Barcelona), Spain \\ ${ }^{*}$ Corresponding author: juergen.eschner@icfo.es
}

Compiled November 1, 2018

\begin{abstract}
We present a tunable, frequency-stabilized, narrow-bandwidth source of frequency-degenerate, entangled photon pairs. The source is based on spontaneous parametric downconversion (SPDC) in periodically-poled $\mathrm{KTiOPO}_{4}$ (PPKTP). Its wavelength can be stabilized to 850 or $854 \mathrm{~nm}$, thus allowing to address two D-P transitions in ${ }^{40} \mathrm{Ca}^{+}$ions. Its output bandwidth of $22 \mathrm{MHz}$ coincides with the absorption bandwidth of the calcium ions. Its spectral power density is 1.0 generated pairs/(s $\mathrm{MHz} \mathrm{mW})$.

(C) 2018 Optical Society of America

OCIS codes: 000.0000, 999.9999.
\end{abstract}

Entangled photon pairs have become an important resource in experiments on fundamental quantum mechanics [1], as well as in quantum communication [2], quantum computing [3], and quantum networks [4]. The best controlled and most widely applied method to create photonic entanglement is spontaneous parametric downconversion (SPDC) in nonlinear crystals [5]. Due to the weak phase matching conditions in small size crystals, these sources usually produce rather broad output spectra with widths on the order of THz. For many applications this is convenient, since the photons interact only with detectors which are not energy selective on this scale. In recent years new SPDC sources have been developed also for narrow band applications [6-8], which are mostly aiming at coupling photonic and atomic systems $[9,10]$. Still, the reported bandwidths are rather broad $(\sim 100 \mathrm{GHz})[6,7]$ compared to atomic transitions, or the sources emit into multiple frequency modes [8].

In this letter we report on a photon pair source designed to permit resonant interaction with single trapped ions [11]. The photon frequency and bandwidth are made to match the linewidth of the $\mathrm{D}_{3 / 2}-\mathrm{P}_{3 / 2}$ and $\mathrm{D}_{5 / 2}-\mathrm{P}_{3 / 2}$ optical transitions in ${ }^{40} \mathrm{Ca}^{+}$, which are centered at 849.8 and $854.2 \mathrm{~nm}$ and have a width of $\sim 20 \mathrm{MHz}$. As prerequisite for efficient coupling, we achieve tunability over this range, a bandwidth reduction of six orders of magnitude compared to standard SPDC sources, and suitable frequency stabilization. This novel source will allow us to perform experiments on the coupling between single quantum systems of light and matter, with applications in quantum networks.

We obtain these characteristics by optimizing the SPDC efficiency via quasi-phase matching [12], by reducing the photon pair bandwidth in a Fabry-Perot cavity filter line, and by stabilizing the absolute photon pair frequency via active feedback on all critical elements in the set-up.

The experimental setup is sketched in Figure 1. The master laser, an extended-cavity diode laser (Toptica
DL-100), provides about $30 \mathrm{~mW}$ of light, tunable between 850 and $854 \mathrm{~nm}$. About $90 \%$ of it is amplified in a tapered amplifier (Toptica TA-100) to $600 \mathrm{~mW}$ output power. The remaining light from the diode is used to stabilize its frequency to a Fabry-Perot cavity of finesse 1000 and $1 \mathrm{MHz}$ linewidth, using the Pound-Drever-Hall $(\mathrm{PDH})$ technique. The length of this cavity is locked to a laser at $852 \mathrm{~nm}$, which itself is stabilized to the D2 line of cesium by saturation spectroscopy. An acousto-optic modulator $(\mathrm{AOM})$ in the $852 \mathrm{~nm}$ laser beam provides fine frequency tuning. This transfer lock technique yields about $125 \mathrm{kHz}$ absolute frequency stability of the master laser [13].

The amplified master beam is frequency-doubled in a lithium tri-borate (LBO) crystal within a bow-tie cavity (Toptica SHG-110), resonant with the master laser wavelength for pump power enhancement, which produces around $100 \mathrm{~mW}$ of 425-427 nm second-harmonic light. The resulting blue beam is sent through a single mode (SM) fiber for mode cleaning and focussed into a second nonlinear crystal where photon pairs are created via SPDC. This crystal is a flux-grown periodically poled $\mathrm{KTiOPO}_{4}(\mathrm{PPKTP})$ crystal of dimensions $20 \times 6 \times 1$ $\mathrm{mm}$ [14]. It has two independent parallel poling gratings imprinted, with periods of 14.03 and $14.63 \mu \mathrm{m}$, for collinear type-II quasi-phase-matching at a temperature of $25^{\circ} \mathrm{C}$ for 849.8 and $854.2 \mathrm{~nm}$, respectively [15].

To optimize the focus of the pump mode, we utilize the reverse process of second-harmonic generation (SHG), which is legitimate due to the narrow-bandwidth of the source [16]. The optimum focussing parameter, the ratio of crystal length and Rayleigh range $\xi=L / z_{R}=5.68$ [12], requires a pump beam waist of $w_{0}=16.1 \mu \mathrm{m}$. Fine tuning and stability of the central wavelength of the photon pairs is achieved by temperature control. The temperature of the crystal is actively stabilized with better than $10 \mathrm{mK}$ precision, and a central wavelength variation of $0.034 \mathrm{~nm} / \mathrm{K}$ was measured.

The generated photon pairs are collimated and split by 


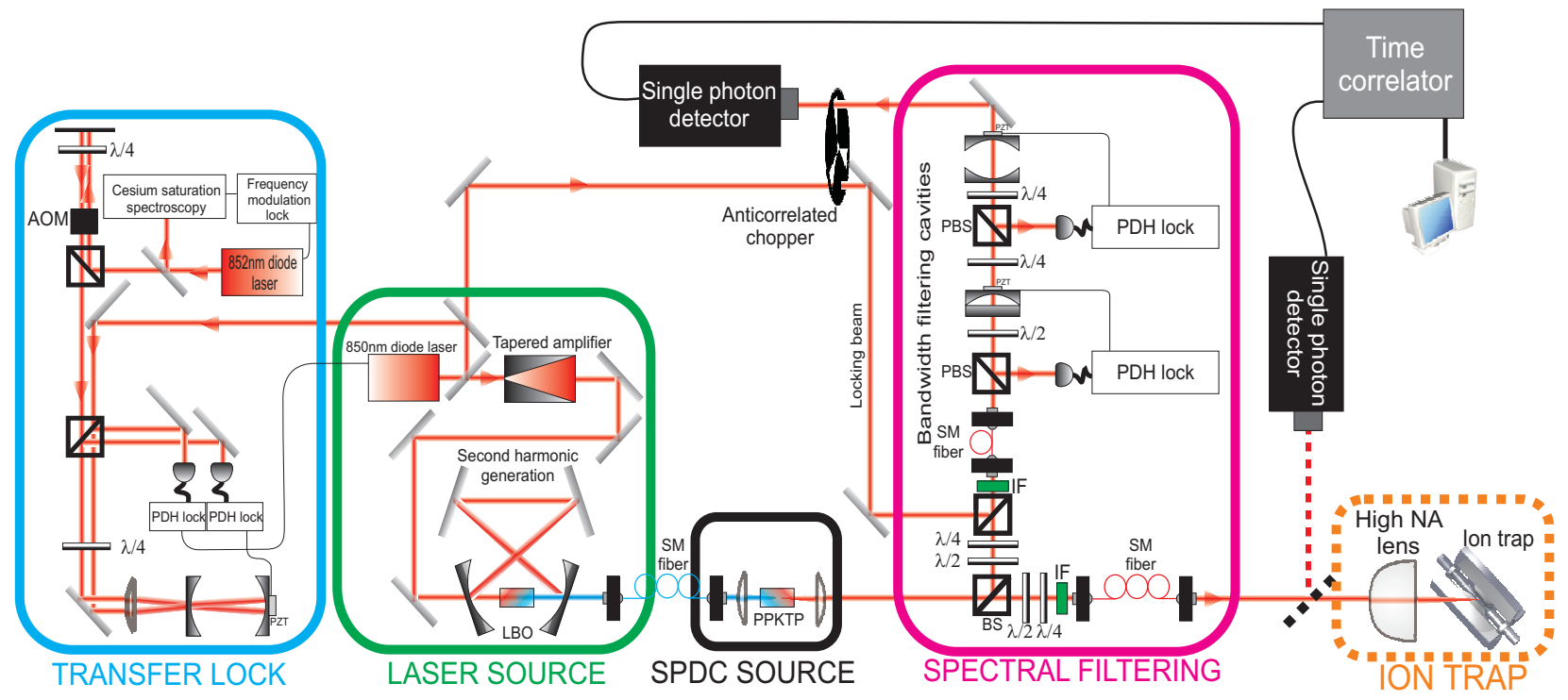

Fig. 1. Scheme of the experimental setup: The master laser is stabilized to an atomic line (transfer lock), amplified and frequency doubled (laser source). The doubled light is down-converted in a type II phase-matched PPKTP crystal (SPDC source). The photon pairs are split and fiber-coupled; one of the photons is spectrally filtered (spectral filtering). The time correlator detects the coincident pairs. Later, the unfiltered photon will be sent to a single ion experiment. Symbols are explained in the text.

a non-polarizing or polarizing beam splitter (BS or PBS), depending on whether their polarization entanglement shall be exploited or not. In order to perform the tomographic reconstruction of the photon pair polarization state, a set of quarter and half-waveplates and a PBS are placed in each arm. Finally, after passing optical bandpass filters (IF) (Semrock FF01-440/40-25), the output modes of the beam splitter are coupled into polarization maintaining single-mode fibers. The single-photon coupling efficiencies are on the order of $42 \%$. The spectral width of the fiber coupled photon pairs was measured to be $143 \pm 4 \mathrm{GHz}$.

After passing through the fiber, the transmission mode of the beam splitter is coupled into a filtering line for bandwidth reduction of the photon pairs. It consists of two Fabry-Perot cavities placed in cascade, designed to cover the whole crystal output spectrum and to provide a single narrow transmission window of the desired bandwidth. Each cavity consists of two high-reflectivity mirrors (Layertec) with a measured finesse of around 620 . The first cavity of $77.5 \mu \mathrm{m}$ length has $3.7 \mathrm{GHz}$ transmission bandwidth, the second cavity of $10 \mathrm{~mm}$ length sets the final filter bandwidth of $\sim 25 \mathrm{MHz}$. Each cavity has a measured transmission, on resonance, of $88 \%$. Together with $42 \%$ fiber coupling efficiency and $45 \%$ detector efficiency, the overall photon detection probability in the filtered arm amounts to $\approx 15 \%$. .

Both cavities are individually stabilized to the master laser wavelength $(850$ or $854 \mathrm{~nm}$ ), and therefore to the desired D-P transition in ${ }^{40} \mathrm{Ca}^{+}$, by means of an auxiliary beam using again the PDH technique. Conservation of energy in the downconversion process together with the narrow frequency bandwidth of the master laser en- sures that, if a photon is transmitted through the filtering line, its partner photon will be on resonance with the atomic transition.

Photons are detected by fiber-coupled avalanche photodiodes (Perkin Elmer SPCM-AQR-15) with 45\% quantum efficiency, $<50$ dark counts/s, and $<1$ ns time resolution. The TTL detection pulses are sent to a correlation electronics module (Picoquant Picoharp 300) which is used primarily as a time discriminator to measure the delay between the pulses in two channels. The data are analyzed with an integrated control software based on LabviewV8.5, which furthermore controls all input and output parameters of the experiment. This allows to remotely run complete measurement series like a full state tomography protocol.

For characterizing the spectral properties of the narrowband pair photon source, a temporal correlation measurement was performed. The photon pairs were split on a polarization beam splitter and detected after filtering in one of the arms. The distribution of the time delays between photons in the two arms is shown in Fig. 2. A peak is resolved which originates from the correlated photon pairs. It shows a characteristic exponential decay due to the filter cavity ring-down. From the decay time we deduce the photon pair bandwidth to be $22.4 \pm 0.5 \mathrm{MHz}$, in agreement with the measured filter cavity linewidth. The background is caused by accidental coincidences of photons with lost partners.

The brightness of the source is obtained from the number of counts in the peak, measured while varying the pump power. The measured rate is 4.8 pairs $/(\mathrm{s} \mathrm{mW})$. For the maximum pump power of $70 \mathrm{~mW}$ this results in an extrapolated detection rate of 340 pairs/s [18]. Taking 


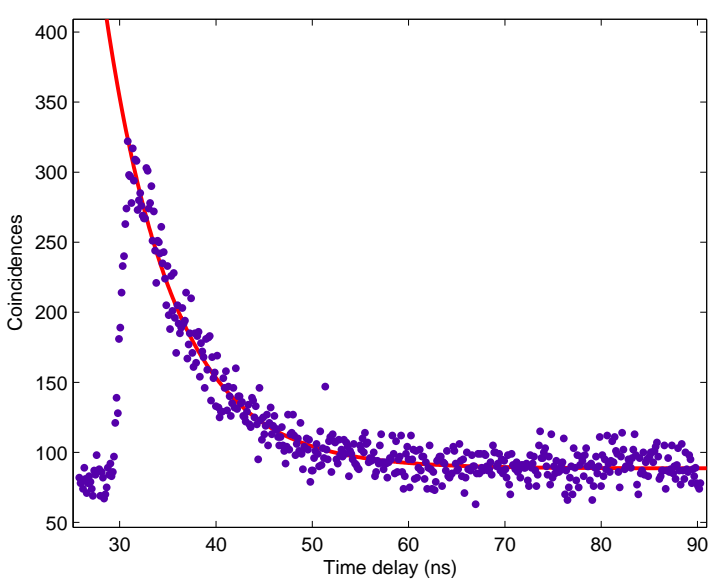

Fig. 2. Time correlation between photons in the filtered and the unfiltered arm (points), and exponential fit (line). The time origin is shifted by an electronic delay.

into account the bandwidth and the detector efficiencies, we find the spectral brightness of generated narrowband pairs to be $1.0 /(\mathrm{s} \mathrm{MHz} \mathrm{mW})$.

To explore the polarization entanglement of the photon pairs, they are split by a 50/50 non-polarizing beam splitter and analyzed by polarization state analyzers in front of the fiber couplers. We perform a full polarization state tomography measurement following [19]. After subtracting the accidental coincidences, we reconstruct the pair photon density matrix $\rho$, as shown in Fig. 3. As a measure of the entanglement quality we calculate the concurrence $C=0.948 \pm 0.015$. The overlap fidelity $F=\left\langle\Psi^{-}|\rho| \Psi^{-}\right\rangle$with the maximally entangled singlet state $\left|\Psi^{-}\right\rangle=\frac{1}{\sqrt{2}}(|\mathrm{H}\rangle|\mathrm{V}\rangle-|\mathrm{V}\rangle|\mathrm{H}\rangle)$, amounts to $0.976 \pm 0.011$. Another practical figure-of-merit are the visibilities of the polarization anti-correlations in the horizontal-vertical and the $\pm 45^{\circ}$ polarization bases, which we find to be $V_{H V}=99.1 \pm 0.9 \%$ and $V_{ \pm}=$ $97.5 \pm 0.9 \%$.
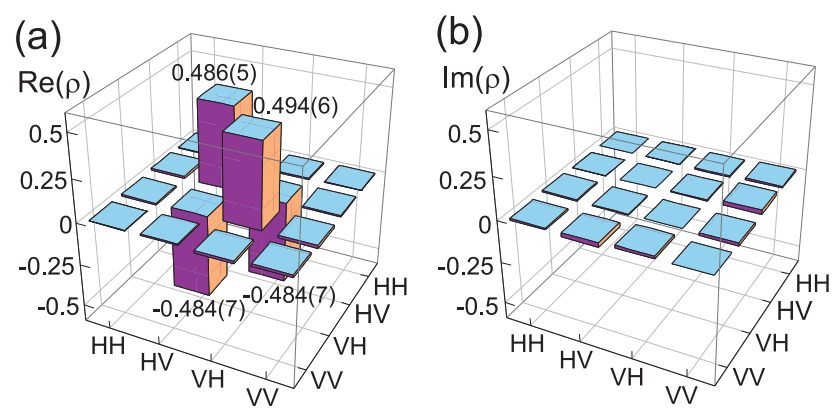

Fig. 3. (a) Real and (b) imaginary part of the polarization state density matrix $\rho$ of the photon pairs in the horizontal-vertical $(\mathrm{H}-\mathrm{V})$ polarization basis.

In conclusion, we have set-up and characterized a narrow-bandwidth, tunable, high spectral density photon pair source, which will allow for efficient coupling to single trapped $\mathrm{Ca}^{+}$ions. The temporal correlation of the photon pairs permits studying the interaction of single atoms with heralded single photons. Exploiting the high-purity entanglement of the source, the coupling of photonic and atomic qubit pairs will be feasible. Prospective experimental applications are entanglement distribution [10] and quantum repeaters [20].

This work has been partially supported by the European Commission (SCALA, contract 015714; EMALI, MRTN-CT-2006-035369), by the Spanish MEC (QOIT, CSD2006-00019; QLIQS, FIS2005-08257; QNLP, FIS2007-66944; FLUCMEM, FIS2005-03394; ILUMA, FIS2008-01051), and by the Generalitat de Catalunya (2005SGR00189). A.H. acknowledges support by the 'Juan de la Cierva' and N.P. by the FPU fellowship programme of the Spanish MEC.

\section{References}

1. Z. Y. Ou and L. Mandel, "Violation of Bell's Inequality and Classical Probability in a Two-Photon Correlation Experiment," Phys. Rev. Lett. 61, 50 (1988).

2. D. Bouwmeester, J. W. Pan, K. Mattle, M. Eibl, H. Weinfurter, and A. Zeilinger, "Experimental Entanglement Swapping: Entangling Photons That Never Interacted," Nature 390, 575 (1997).

3. E. Knill, R. Laflamme, and G. Milburn, "A scheme for efficient quantum computation with linear optics," Nature 409, 46 (2001).

4. J. I. Cirac, P. Zoller, H. J. Kimble, and H. Mabuchi, "Quantum State Transfer and Entanglement Distribution among Distant Nodes in a Quantum Network," Phys. Rev. Lett. 78, 3221 (1997).

5. P. G. Kwiat, K. Mattle, H. Weinfurter, A. Zeilinger, A. V. Sergienko, and Y. Shih, "New High-Intensity Source of Polarization-Entangled Photon Pairs," Phys. Rev. Lett. 75, 4337 (1995).

6. F. Knig, E. J. Mason, F. N. C. Wong, and M. A. Albota, "Efficient and spectrally bright source of polarizationentangled photons," Phys. Rev. A 71, 033805 (2005).

7. A. Fedrizzi, T. Herbst, A. Poppe, T. Jennewein, and A. Zeilinger, "A wavelength-tunable fiber-coupled source of narrowband entangled photons," Opt. Exp. 15, 15377 (2007).

8. C. E. Kuklewicz, F. N. C. Wong, and J. H. Shapiro, "Time-Bin-Modulated Biphotons from CavityEnhanced Down-Conversion," Phys. Rev. Lett. 97, 223601 (2006).

9. A. D. Boozer, A. Boca, R. Miller, T. E. Northup, and H. J. Kimble, "Reversible State Transfer between Light and a Single Trapped Atom," Phys. Rev. Lett. 98, 193601 (2007).

10. B. Kraus and J. I. Cirac, "Discrete Entanglement Distribution with Squeezed Light," Phys. Rev. Lett. 92, 013602 (2004).

11. S. Gerber, D. Rotter, M. Hennrich, R. Blatt, F. Rohde, C. Schuck, M. Almendros, R. Gehr, F. Dubin, J. Eschner, in preparation.

12. R. W. Boyd, Nonlinear Optics, Academic press (2003).

13. F. Rohde, private communication. 
14. The crystal was fabricated by C. Canalias from the Laser physics department of the KTH in Stockholm.

15. K. Kato and E. Takaoka, "Sellmeier and Thermo-Optic Dispersion Formulas for KTP," Appl. Opt. 41, 5040 (2002).

16. M. W. Mitchell, "Parametric down-conversion from a wave-equations approach: geometry and absolute brightness," arXiv/0807.3533 (2008).

17. R. W. Boyd and D. A. Kleinmann, "Parametric Interaction of Focused Gaussian Light Beams," J. Appl. Phys. 39, 3597 (1968).

18. We could not measure this rate directly, due to saturation of the detector in the unfiltered arm.

19. D. F. V. James, P. G. Kwiat, W. J. Munro, and A. G. White, "Measurement of qubits," Phys. Rev. A, 64, 052312 (2001).

20. H.-J. Briegel, W. Dür, J. I. Cirac, and P. Zoller, "Quantum Repeaters: The Role of Imperfect Local Operations in Quantum Communication," Phys. Rev. Lett. 81, 5932 (1998). 